\title{
SISTEMATIZACIÓN DE LOS ARTÍCULOS DE GRADO DE LAS ESPECIALIZACIONES EN COMUNICACIÓN DE LA UAC
}

\section{SYSTEMATIZATION OF THE DEGREE WORKS OF THE SPECIALIZATIONS IN COMMUNICATION IN THE UAC}

Javier Rosero Molina. Universidad Autónoma del Caribe. Colombia

Darvis Rodríguez Chaparro. Universidad Autónoma del Caribe. Colombia

Carlos Ramos Maldonado. Universidad Autónoma del Caribe. Colombia

\section{RESUMEN}

Los artículos de revisión, reflexión e investigación hacen parte de los requisitos de grado para los estudiantes de las especializaciones en Comunicación de la Universidad Autónoma del Caribe. Teniendo en cuenta el firme propósito del aseguramiento de la calidad de ambas especializaciones se hace necesario la sistematización y el análisis de los trabajos de grados, el ejercicio nos permite establecer con precisión las temáticas más y menos utilizadas, y los resultados serán pieza clave en aras de aportar a la elaboración de nuevos proyectos de grado y la contribución a la estructuración e implementación de nuevos contenidos curriculares definidos dentro de los hilos conceptuales en Gerencia de la Comunicación Organizacional y Gerencia de la Comunicación para el Desarrollo Social. Para la sistematización y análisis de los trabajos de grado se empleará el software estadístico para las ciencias sociales y humanas SPSS. A partir de ello, se identificaron áreas sobre las cuales es prudente profundizar con la intención de que se mejoren, como es la búsqueda constante y la calidad de la educación ofrecida en estos posgrados.

PALABRAS CLAVE: comunicación organizacional, desarrollo social, especialización, gerencia, sistematización, trabajos de grado.

\section{ABSTRACT}

Review, reflection and research articles are part of the degree requirements for students of the Communication specializations of the Universidad Autónoma del Caribe. Taking into account the firm purpose of the quality assurance of both specializations, it is necessary to systematize and analyze the degree projects, the exercise allows us to establish precisely the most and least used topics, and the results will be a key piece for the sake of to contribute to the development of new degree projects and the contribution to the structuring and implementation of new curricular contents defined within the conceptual threads in Organizational Communication Management and Communication Management for Social Development. For the systematization and analysis of the degree projects, the statistical software for the social and human sciences SPSS will be used. From this, areas were identified on which it is prudent to go deeper with the intention of improving them, such as the constant search and quality of the education offered in these postgraduate courses. 
KEYWORDS: degree works, management, specialization, systematization, organizational communication, social development.

\section{Cómo citar el artículo:}

Rosero Molina, J., Rodríguez Chaparro, D. y Ramos Maldonado, C. (2020). Sistematización de los artículos de grado de las especializaciones en comunicación de la UAC. Revista de Ciencias de la Comunicación e Información, 25(3), 57-70. doi: http://doi.org/10.35742/rcci.2020.25(3).57-70

\section{INTRODUCCIÓN}

Los programas de Especialización en Gerencia de la Comunicación Organizacional y Gerencia de la Comunicación para el Desarrollo Social, activados en el año 2011, han sido escenarios para fomentar la investigación dentro de la Facultad de Ciencias Sociales y Humanas-FCSH- de la Universidad Autónoma del Caribe (UAC). A partir de la implementación de la modalidad de trabajo de grado como etapa final del proceso de cursada de cada posgrado, se han producido decenas de artículos de investigación.

Sin embargo, considerando el actual estado de convergencia digital que atraviesa la moderna sociedad de la información y del conocimiento, es indispensable la integración de redes sociales al igual que dispositivos móviles en la cotidianidad, especialmente en el fluctuante contexto socio-cultural del Colombia, por lo cual este trabajo se propone sistematizar los artículos de grado en aras de crear nuevas estrategias pedagógicas que permitan facilitar información para apoyar otras producciones académicas y realizar modificaciones a los Syllabus respectivos.

Por lo tanto, se hace necesario considerar cuál es el estado actual de las investigaciones, así como la formación en Comunicación Social tanto en Colombia como en América Latina, para comprender sus variaciones y particularidades, las cuales fundamenten el marco de análisis que se implementarán para la sistematización de los trabajos de grado en las Especializaciones en Comunicación de la UAC. Con base en esto, es imperativo entender cómo se ha desarrollado la formación en comunicación en dicha universidad en la última década. Beltrán (2016) plantea la necesidad de estrechar los vínculos existentes entre docencia e investigación, en aras de que se pueda entonces desarrollar una aproximación a la realidad más profunda y a partir de la cual se puedan re-evaluar las áreas de actuación de tanto alumnos como docentes.

Esto no es un aporte menor, dado que actualmente dentro de la UAC existe una modalidad de articulación de los pregrados de la FCSH con las Especializaciones de la misma área, por lo que resulta pertinente considerar el tipo de formación que están teniendo los estudiantes de cara su vinculación a los posgrados gracias a esta posibilidad. A partir de reflexiones al respecto (Gonzales y Rosero, 2015), los programas de Comunicación en Colombia requieren una óptica centrada en aspectos sociales y participativos, pero la cual también reconozca la importancia de las organizaciones en la estructuración del tejido social. Así, se denota la necesidad 
de revisar cómo los estudiantes, una vez están a punto de egresar, están articulados con nociones como estas o si están desconectados de la misma. A su vez, esta investigación funciona como diagnóstico para comprender la forma en que los estudiantes de posgrado de la $\mathrm{FCSH}$, se conectan con el contexto inmediato y necesidades respectivas.

No obstante, dichas condiciones deben ser exploradas dependiendo de cuál de las dos especializaciones y qué trabajos respectivos se están observando. Con respecto al aspecto organizacional, de acuerdo con Piñeros (2018), existe una falta de interés por cómo se ha venido desarrollando al abordaje de esta área de la comunicación desde los pregrados del país:

Por otra parte, existe poca regularidad en el número de investigaciones producidas de acuerdo a los años referenciados, lo que demuestra un creciente desinterés por la comunicación organizacional como objeto de investigación, viéndose seriamente afectada la proyección epistemológica de la profesión en un momento en el que demanda fundamentos científicos para consolidar su estatus, generados desde las facultades de comunicación ya que los aportes que provengan de otras disciplinas estarán sesgados hacia sus propias epistemologías. (p.64).

No es un factor para tomar con ligereza, la diversidad de abordajes en la cual los estudiantes enfocan sus trabajos de grado, es un indicador sobre a qué área se están interesando como egresados, generando impacto en la sociedad. Si bien la Especialización en Gerencia de la Comunicación Organizacional cuenta con un mayor número de producción de artículos de grado, productos de investigación consecuentes, es pertinente revisar, categorizar y entender a partir de los mismos, el aporte que los resultados pueden generar para la reflexión o renovación de los debates necesarios para mantener vigente al posgrado. Se estima encontrar posibles ejes conceptuales entre todos los textos que se han aprobado como proyectos de grado, por lo que se pretende a su vez considerar cómo se compenetran con las condiciones del entorno del tiempo en que fueron producidos.

En este orden de ideas, estos artículos al ser productos de investigaciones puntuales ponen un especial énfasis en el trabajo de campo empírico en comunicación, lo cual podría arrojar una mayor proximidad con el entorno inmediato y por lo tanto el contexto.

El levantamiento es un estudio descriptivo, con énfasis en la recolección y sistematización de los datos empíricos para utilización práctica, en tanto la investigación social parte de la fundamentación empírica de los datos para contribuir al cuerpo del conocimiento teórico y metodológico de un determinado campo de estudios, independientemente de su utilización inmediata. Esta es, a mi modo de ver la condición que debe regir la investigación académica de Comunicación, pues solamente a través de la elaboración interpretativa de los datos se puede lograr un patrón de trabajo científico en el campo de la Comunicación (Vasallo de Lopes, 1999, p.10).

A su vez, esto se hace más necesario al considerar el enfoque de la segunda especialización, el cual es en Desarrollo Social. Este concepto que si bien se ha desarrollado desde los años 80 (Gumucio-Dagron, 2011), se concibe con una 
alternativa de comunicación en la cual se le conceptualiza como un proceso de creación de vínculos y sentidos, orientados a que los medios de comunicación estén al servicio de las necesidades de las comunidades dentro un contexto cultural con complejidades, siendo el comunicador un gestor, más que protagonista. Esto implica un énfasis particular en las experiencias y como a partir de estas se fortalece el pensar conceptual (Cadavid Bringe y Gumucio-Dagron, 2014), puede conducir a una práctica en comunicación con mayor sintonía con el contexto sociocultural actual. Pero, como sucede con su especialización hermana, esto debe verificarse con la sistematización de los trabajos de grado, si se quiere contar con un fundamento adecuado al respecto.

Ahora bien, si se quiere hablar de una posible renovación curricular, o más bien, nutrirla con los resultados e interpretaciones de esta investigación, es muy prematuro todavía plantear una propuesta al respecto. Por lo tanto, se hace necesario revisar los mismos fundamentos de todo el plan de estudio, en aras de contar con una relación intersubjetiva con los eventuales resultados de esta investigación, lo cual puede conducir a una interpretación apegada al contexto y sobre la cual se construya un proceso educativo pragmático.

\section{OBJETIVOS}

\subsection{Objetivo General:}

Sistematizar los trabajos de grado de las Especializaciones en Comunicación de la UAC en aras de que contribuyan a algunos aportes para nuevos trabajos de grado y a la fundamentación para realizar modificaciones en los contenidos curriculares de las mismas en el marco de la actual sociedad de la información y el conocimiento.

\subsection{Objetivos Específicos}

Examinar los trabajos de grado de las Especializaciones en Comunicación de la UAC en aras de identificar hilos conceptuales frecuentes.

Establecer categorías en las cuales se enmarcan los trabajos de grado de las Especializaciones en Comunicación de la UAC relacionándolas con los contenidos curriculares de las mismas

Desarrollar propuestas para transformar o modificar los actuales Syllabus de los Módulos que componen la malla curricular que componen las Especializaciones en Comunicación de la UAC

\section{MARCO REFERENCIAL}

Acudir a trabajos de grado profesional, tesis, artículos científicos, citas o referencias bibliográficas ya elaboradas como fuentes documentales para una nueva investigación, más allá de la práctica de campo o estudio de caso, forja el mapeo teórico e historial del nuevo proceso investigativo que se emprende y, de paso, permite revisar el estado microcurricular de los programas académicos. Es decir, se establece entonces una relación iniciática y complementaria entre la necesidad epistemológica de la investigación, que se puede encontrar en otros trabajos 
similares, y las acciones de campo, ilación teórica que acompaña el desarrollo del nuevo paper en todas sus etapas, más la posibilidad del rediseño curricular de los programas académicos.

Tras un diseño de investigación, el gestor recaba documentalmente el material producido por otros autores sobre el tema a indagar y precisa clasificar un cuadro de ideaciones temáticas con palabras o frases nucleares (clave) que lo lleva a revisar algunos textos con enfoques confiables y otras experiencias muy útiles para su nueva producción, en este caso, ya sea para crear contenidos de revisión bibliográfica, de reflexión o de resultado de investigación, o sea para actualizar un Syllabus académico.

Aunque ninguna teoría es absolutamente verdadera (Popper, 1989), textos científicos retrospectivos, citas o referencias bibliográficas permiten acercar al investigador a un criterio de significación sobre su objeto a investigar.

Acudir a fuentes documentales para alimentar un proceso nuevo no es quitarle originalidad al mismo, por el contrario, es, por un lado, demostrar la honradez del investigador y, por el otro, legitimar el resultado con aportes certeros ya divulgados por personas mejores conocedores del tema o que han procesado información que no es necesario construir como nuevo, concurriendo a un enfoque socio-crítico para abordar los mecanismos de reflexión e interpretación, dando, por supuesto, el crédito correspondiente.

Villaseñor (2008), acudiendo a Krummel (1993), recomienda al investigador confeccionar un repertorio temático que pueda servirle de referencia o permita algunas pistas en la cadena bibliográfica para recuperar información lo más original posible. Dado que el trabajo bibliográfico es abundante tanto en el mundo físico como virtual, y que reducirlo a contenidos de interés específico es dispendioso, se debe asistir a la selección solidaria instituciones/individuo sobre documentación y compilación temática, de tal manera que logre encerrarse exponencialmente el sello referencial.

Esta fase de documentación temática pertinente es importante fundamentalmente para la teorización y la concesión de una idea clara y distinta del problema de investigación y la delimitación del objeto de estudio, ya que si existe una diversidad de autores sobre un mismo tema que conlleva a tensiones dialécticas, implicando, por consiguiente, diferentes lógicas de pensamiento, racionalidades, discursos y distintos respaldos epistemológicos, de acuerdo a circunstancias y entornos culturales, entonces se debe comparar, excluir, incorporar o complementar los saberes retrospectivos con el que se pretende construir conocimiento nuevo.

Entonces, es recomendable que para la organización de dicho repertorio temático, se defina primero un reservorio impreso o digital de textos que sirvan de referencia para albergar consultar y orientar información de interés en vía al desarrollo de un trabajo de investigación determinado, y luego, ya clasificado ese material en el anaquel particular, se sistematice en un modelo instruccional segmentado que facilite la labor del nuevo investigador. 
Para Pérez (2016), sistematizar información es clasificar, ordenar y clarificar un proceso de recolección de datos respecto a una temática realizada que incluye títulos/palabras clave/autores/referencias bibliográficas, etc. Se trata de un compendio complejo, que involucra una relación de identificaciones, antecedentes y testimonios básicos para apoyar y/o soportar un trabajo nuevo.

Para el proceso de sistematización, es necesario recurrir a todo tipo de información y documentación provenientes de fuentes impresas, digitales, orales, visuales, vivenciales, y otras, disponibles en la institución, organización o en la comunidad que auspicia o alberga la experiencia. Por ello, es significativo recuperar informes, diagnósticos, reportes técnicos, planes operativos, memorias y cuenta, datos estadísticos, notas y apuntes, fotografías, videos, periódicos, datos personales de los involucrados, realizar entrevistas, mantener conversaciones con los involucrados. Asimismo, es importante incorporar bibliografía general y materiales relacionados con el tema específico de la experiencia, entre otros (Pérez, p.38).

Toda esta datación codificada debe ser procesual para la facilidad de conseguir información en ambiente amigable y con claridad referencial e ideacionaria en la definición de contenidos teóricos y epistemológicos.

Este empoderamiento proviene de la "reflexividad dialógica", propia de la sistematización. Tal como lo expone Ghiso (2011), el primer impulso es seguir las rutinas de la investigación tradicional, con un marco de teorías preconcebida. El objeto que persigue la sistematización establece la necesidad de encontrar otras rutas posibles, en las que antes de adoptar una teoría o un concepto, podamos establecer diálogos con nosotros mismos y con los otros, reconociendo diferentes posturas y construcciones cargadas de objetividad y subjetividad (Pérez, p.18).

Las sistematizaciones bibliográficas, documentales o de "papers", de acuerdo con el caso que ocupa esta ponencia, son de tres clases:

1. Totales, si engloba toda la información bibliográfica sobre un tema determinado, propio del ejercicio de las bibliotecas, hemerotecas o dependencias afines, generalmente académicas.

2. Parciales, si se reduce a un tipo de trabajo documental, por ejemplo, tesis de grado o artículos científicos, que incluye ejes temáticos/títulos/autores/fechas y referencias bibliográficas, diligenciadas por una dependencia académica o grupo de investigación.

3. Interna, cuando se procesa la información que contiene un solo documento, haciendo uso de ejes temáticos, tópicos, citas bibliográficas y palabras clave ${ }^{1}$, entre otros aspectos, que procesa el investigador.

Ahora bien, la opción grado (PEP, 2016) de las especializaciones en Comunicación Social de la Universidad Autónoma del Caribe, tanto en la mención Gerencia de la Comunicación Organizacional como en la de Gerencia de la Comunicación para el Desarrollo Social es la realización de "paper" o artículo científico publicable, los

\footnotetext{
1 Unidades nucleares sintácticas mínimas de clasificación léxico/semántica y semántico/pragmática o sus derivaciones y dispersiones o recurrencias, que recogen las propiedades internas del discurso básico.
} 
estudiantes y egresados no graduados que están en esta fase final requieren información disponible sobre trabajos ya aprobados para grado, de tal manera que les sirva de marco referencial bibliográfico, temático, teórico o experimental para su nueva producción; es decir, estas producciones tienen un impacto académico que tiene que ver con la apropiación que se realiza del conocimiento elaborado en otros trabajos de investigación, por lo que sistematizar los trabajos de grado ya sentenciados positivamente por la Dependencia es de valor preciado.

De igual manera, estos mismos trabajados de grado en su revisión sistematizada sirven para alimentar la actualización o modificación de Syllabus de programas académicos, o elaborar propuestas nuevas de microcurrículos, de acuerdo con las innovaciones teóricas o experimentales que se vayan mostrando en dichos trabajos.

Un paper es un trabajo académico de comunicación científica aprobado y publicable en revista indexada, de acuerdo a normas y estructura preestablecidas por la Dependencia, cuyo objetivo es producir conocimiento nuevo exponencial sobre el área del saber en comento, ya sea por revisión bibliográfica, reflexión sobre textos publicados 0 experiencias demostradas, o sobre resultado de investigación realizada, que pueda convertirse en obra de consulta profesional o sirva para el desarrollo de métodos experimentales innovadores, lo que procura suprimir las barreras entre la producción del conocimiento y el aprovechamiento del mismo por la sociedad.

Según Ramírez, Martínez y Castellanos:

Cuando la investigación y los conocimientos generados, difundidos y divulgados a partir de esta, provenientes de una institución o una alianza de organizaciones, se orientan al objetivo de suplir necesidades de la sociedad o son utilizados indirectamente en desarrollos puntuales para su beneficio, puede hablarse de impacto social o apropiación social del conocimiento científico. La responsabilidad social de las instituciones académicas se convierte en un compromiso consciente y ético con los actores con los que se relacionan (2012, p.36-37).

$\mathrm{Y}$, precisamente, sistematizar dicho reservorio como recursos para sintetizar contenidos aplicables a investigaciones específicas es una dinámica que se ofrece en el presente instrumento como infraestructura codificada con la finalidad de facilitar información bibliográfica y teórica, más experiencias documentadas, en lo que respecta a la elaboración de trabajos finales de grado de las Especializaciones en Comunicación Social de la Universidad Autónoma del Caribe.

\section{METOdOLOGÍA}

La presente investigación es de carácter cuantitativo, los estudios que utilizan este enfoque confían en la medición numérica, el conteo y en uso de estadística para establecer indicadores exactos (Hernández, Fernández y Baptista, 2015). El diseño está estrechamente relacionado al estudio de caso referente a las especializaciones en Gerencia de la comunicación Organizacional y Gerencia de la Comunicación para el Desarrollo social de la Universidad Autónoma del Caribe, buscando determinar la fuerza de asociación o correlación entre variables, la generalización y objetivación a través de una muestra para ser inferencia a una población (Pita Fernández y 
Pértegas Díaz, 2002) por ello se establecen variables y se toma como muestra los artículos (revisión - reflexión - investigación) realizados por los estudiantes de ambas especializaciones desde el año 2015 al primer trimestre de 2020.

En este sentido, también se tiene contemplada la implementación de criterios de investigación cualitativa, dado que es prudente analizar los aspectos interpretativos y las particularidades (Hernández, Fernández y Baptista, 2015) de cada artículo en aras de producir una aproximación más certera al contenido, funcionando a su vez como complementación a los datos cuantitativos previamente establecidos. De ahí qué si bien este enfoque es tomado en cuenta, así como aplicado, no tiene la suficiente presencia dentro de esta investigación para calificarla como mixta. Siendo una investigación en comunicación, la complejidad (Múnera, 2010; Cadavid Bringe y Gumucio-Dagron, 2014), demanda un abordaje flexible a factores involucrados, reafirmando entonces el valor de criterios cualitativos aún dentro de una investigación primariamente cuantitativa.

Con respecto a la población de esta investigación, constituiría los artículos producidos por estudiantes desde 2012 hasta 2020, por lo que se optó por seleccionar un tipo de muestra no probabilística (Hernández, Fernández y Baptista, 2015), compuesta por los artículos producidos desde 2015-01 hasta marzo de 2020. Por este volumen de contenido para explorar, esta investigación sigue en curso.

Así esta investigación se proyecta por etapas. En la primera se procedió con la recopilación y organización de los trabajos de grados de ambas especializaciones, se identificaron los siguientes ejes conceptuales para cada una de estas:

> Gerencia de la Comunicación Organizacional los ejes temáticos establecidos para esta especialización hacen referencia al Clima y cultura organizacional, Comunicación externa e interna, Comunicación y marketing, y finalmente Comunicación y tecnología.

$>$ Gerencia de la Comunicación para el Desarrollo Social. Teniendo en cuenta los diferentes temas elegidos por los estudiantes en esta especialización se establecieron los siguientes ejes temáticos Medios comunitarios, Comunicación y cultura, y finalmente Comunidades minoritarias

En la segunda etapa se realizó la revisión del $50 \%$ de los trabajos de grado teniendo en cuenta las variables que permitían la clasificación de los trabajos, el título planteado por el autor o autores, daba cierta pista de la temática a tratar en cada trabajo, las palabras clave (nucleares), que nos permiten la búsqueda y selección de los artículos dentro de bases de datos extensas, y así mismo también establecen las características del artículo, el contenido estructural para definir a qué tipo de artículo pertenece, el número de páginas para determinar la extensión del trabajo y finalmente la fecha de publicación para ubicarlo en los periodos comprendidos del 2015-01 al 2020-01 (primer trimestre). De allí se revisaron los contenidos particulares de los artículos agrupados en cada eje para identificar particularidades, conexiones y diferencias entre los textos de cada eje, así como también a sus semejantes correspondientes a la Especialización hermana.

Para la tercera etapa se utilizará el software estadístico SPSS para la creación de variables establecidas para el análisis y la sistematización de los trabajos de grado de ambas especializaciones. Se alimentarán cada una de las variables con los 
respectivos registros y posteriormente se establecerá el informe teniendo en cuenta la relación de las variables. Dichas variables se construirían con la información cualitativa extraída en la etapa para contrastar, enriquecer y profundizar en los hallazgos más relevantes.

De esta manera, se establece que el componente que permite esa articulación entre lo cuantitativo y criterios cualitativos, sin transformar el enfoque de toda la investigación a diseño mixto es la perspectiva crítico social. Esta para términos de esta investigación corresponde a:

Esta perspectiva surge como respuesta a las tradiciones positivistas e interpretativas y pretenden superar el reduccionismo de la primera y el conservadurismo de la segunda, admitiendo la posibilidad de una ciencia social que no sea ni puramente empírica ni solo interpretativa. Este paradigma crítico introduce la ideología de forma explícita y la autoreflexión crítica en los procesos del conocimiento. (Yelitza; 2008, p 33)

Con base en esto, se aspira a producir resultados lo más certeros, correspondientes y articulados con el material analizado, así como realizar una propuesta compleja que no se centre solo en un componente o sea fragmentaria.

Es prudente aclarar que la elaboración de artículos ya sea de revisión, reflexión o investigación hace parte de los requisitos para obtener el título de Especialista en Comunicación en cualquiera de los dos posgrados, es por eso qué se trabaja con el producto final de los estudiantes de segundo semestre que desarrollan el curso Humanismo e Investigación que hace parte del pensum de ambas especializaciones. Los alumnos emplean alrededor de 5 meses para realizar su artículo, durante ese proceso cuentan con el acompañamiento del docente a cargo del curso respectivo quien, aparte de los dos encuentros presenciales, se programan tutorías para el seguimiento de los trabajos. A continuación, se muestra la relación del número de artículos analizados hasta el momento.

Tabla 1. Artículos especializaciones comunicación: 2015-2020

\begin{tabular}{|l|c|}
\hline ESPECIALIZACIÓN & NÚMERO DE ARTÍCULOS \\
\hline Gerencia de la Comunicación Organizacional & 61 \\
\hline Gerencia de la Comunicación para el Desarrollo Social & 26 \\
\hline
\end{tabular}

Fuente: Elaboración Propia

Por supuesto, estos no son todos los artículos producidos durante el periodo de tiempo establecido en la investigación, pero es el avance que se ha tenido de cara al volumen y las circunstancias coyunturales que ha traído la emergencia sanitaria que trajo consiguió el COVI-19.

\section{DISCUSIÓN}

Abordando los resultados parciales de la investigación, se observa que, en la muestra de trabajos de grado correspondiente a la Especialización en Gerencia de la Comunicación Organizacional, el eje de Comunicación interna y externa está presente como tópico central en el 35\% de los documentos entregados. Mientras que los ejes de Clima y Cultura Organizacional, así como Comunicación y Marketing, 
figuran, cada uno, como temas principales en $25 \%$ de los textos elaborados por los estudiantes. Finalmente, Comunicación y Tecnología constituye el eje final en los artículos de grado, con el 15\% restante. A partir de ello se denota una tendencia clara de los estudiantes a emprender proyectos en las áreas que competen a la gestión, funcionamiento y dinámicas de la organización.

Por otra parte, los resultados parciales correspondientes a trabajos de grado pertenecientes a la Especialización en Gerencia de la Comunicación para el Desarrollo Social exhiben que el eje rector con mayor presencia en dichos documentos es el de Comunicación y Cultura, con 40\%. En este sentido, Comunidades Minoritarias se encuentra como tópico central en 30\% de los artículos elaborados y finalmente los Medios Comunitarios son el tema central del $25 \%$ de textos restantes. En contraste con las investigaciones realizadas en su posgrado hermano, los estudiantes se enfocan en organizaciones no estructuradas o en grupos sociales con necesidades particulares. No se toma como un hallazgo inesperado, sino consecuente de los enfoques particulares de cada Especialización, sin embargo, la comparación en este caso es potencialmente enriquecedora, en aras de identificar en que se puede aplicar sobre los procesos pedagógicos de cada programa en el otro.

Es prudente clarificar que estos resultados pueden contar con variaciones o es factible que se reflexione sobre los mismos de acuerdo con los resultados finales, contenido de los artículos que restan por analizar, así como en observaciones que se realicen durante el resto del proceso investigativo. Así, cualquier comparación que se haga en esta etapa sobre los resultados parciales entre ambas especializaciones, podría llevar a conclusiones erradas e inconsistentes. Las divergencias se observan, pero las condiciones, situaciones y significaciones que llevaron a su estructuración, requiere un tiempo más amplio para su apropiada examinación, deliberación y articulación de saberes.

En el caso particular de los resultados concernientes a la Especialización en Gerencia de la Comunicación Organizacional, existe una continuidad con la formación en Comunicación "pre-gradual" (Gonzales y Rosero, 2015; Beltrán, 2016) en el término que se identifica una tendencia hacía los aspectos instrumentales o funcionales de la gestión interna de la organización. Por lo que, sería menester considerar diversificar la profundización en diversas sub-áreas del ámbito organizacional. Así surgen varias posibilidades, como ofrecer otras electivas que flexibilicen la comprensión del establecimiento de vínculos y producción de sentido en una organización, las variaciones contextuales y los diversos factores involucrados. En ese orden ideas podrían ser pertinentes más actividades de apropiación social del conocimiento con comunidades, organizaciones y actores externos a la UAC, integrándolos a las discusiones al interior de los módulos, las cuales construyan el interés en los estudiantes a explorar otras áreas dentro de su posgrado.

Ahora bien, en lo que respecta a los resultados correspondientes a los trabajos de grado en la Especialización en Gerencia de la Comunicación para el Desarrollo Social, existe una razón social de enfoque comunitario y gestión descentralizada de los procesos comunicativos (Gumucio-Dagron, 2011), a lo cual se observa hay correspondencia. Pero, surge el cuestionamiento si estas investigaciones responden 
a necesidades objetivadas dentro del contexto socio-cultural en el que se desenvuelven los estudiantes. No es una cuestión menor, dado que la articulación entre contenidos, investigaciones de los estudiantes y actualidad en el entorno, es una trinidad que debe ser balanceada si se espera un proceso educativo coherente. El énfasis mayoritario en Comunicación y Cultura podría ser un indicador benéfico en esta área, aún si no es concluyente.

En ese orden de ideas se identifica un factor común en los temas menos abordados por los trabajos de grado de cada especialización: La tecnología y los medios. Aunque nociones "mediocentristas" (Múnera, 2010; Vasallo de Lopes, 1999) que dan mayor valor a los instrumentos en la formación en comunicación son frecuentes a nivel de pregrado, se evidencia que dentro de los posgrados de la FCSH no son el foco, un posible síntoma de una diversificación de los enfoques prácticos de los estudiantes una vez llegan a un nivel superior de información. No obstante, al revisar cualitativamente los textos, los medios no desaparecen por completo, sino que su rol es complementario en los planteamientos de investigación; así también es el caso con otras herramientas tecnológicas. La minoritaria cantidad de investigaciones resulta interesante considerando la actual sociedad de la información (Unesco, 2012) por lo que los resultados subvierten las expectativas al respecto. Esto conduce al debate de qué tanto énfasis debe tener el uso crítico de tecnologías (incluyendo medios) en los planes de estudio de ambas especializaciones, dado que si bien hay consideración de estas en el P.E.P (2016).

Con base a lo anterior, se interpreta qué a partir de los resultados obtenidos hasta el momento, la necesidad identificada para las 2 especializaciones es adaptarse a las situaciones cambiantes del contexto socio-cultural. Sea considerar implementaciones sobre el uso crítico de medios, tecnologías, así como de factores determinantes en la creación de vínculos significativos al interior de la organización o una crisis macro que puede presentarse inesperadamente, el carácter multi e interdisciplinario de la formación en comunicación (Múnera, 2010), es el componente sobre el cual se pueden fundamentar las modificaciones necesarias para suplir dicha necesidad. Sin embargo, para tener certeza alguna sobre estas falencias, oportunidades para mejorar y renovar, es imperativo concluir esta investigación.

\section{CONCLUSIONES}

En primera instancia, se enfatiza que la investigación todavía se encuentra en desarrollo, por lo que, hasta el momento, se está ad-portas de iniciar lo que se denomina como fase 3 de la revisión de los trabajos de grado, para entonces someter toda la información y datos acumulados al procesamiento. De ahí que, hasta el momento se puede plantear unas tendencias temáticas, como se expuso previamente, las cuales orientan la exploración en conceptual en cada una de las especializaciones.

Así se puede afirmar que el ejercicio realizado hasta el momento fundamenta y permitir decir que se ha cumplido el objetivo general de la investigación concerniente a la sistematización de los trabajos de grado en cuestión. En ese mismo sentido, con base en la examinación de los documentos se encontraron áreas de coincidencia conceptual generales, con las que se hace posible establecer categorías de tanto de análisis, como de direccionamiento, para los artículos 
producidos en los posgrados. Sobre esto último se proyectan ligeros cambios, sin embargo, cabe la posibilidad, de acuerdo con indagaciones preliminares, que los trabajos por considerar alimenten las categorías ya establecidas.

Con base en ello, es prematuro todavía diseñar o desarrollar propuestas sean de modificación de Syllabus o del plan de estudio de cada especialización, pero hay indicios sobre posibles áreas sobre las cuales enfocarse para su mejoramiento. Se evidencia una falta de énfasis en los trabajos de grado con respecto análisis que tome en cuenta tecnología y sus implicaciones actuales, lo cual, dentro de actual Sociedad de la información y el conocimiento (Unesco, 2012), resulta desconcertante, debido a que la adaptación y apropiación de tecnologías, se presume como una ventaja de la generación actual de estudiantes. Esta mínima presencia, en comparación a las categorías parciales actualmente establecidas, invita a un análisis más profundo.

Si bien la investigación está en todavía en desarrollo, se puede apreciar cómo se necesita reconsiderar algunos aspectos en formación dentro de las Especializaciones, como lo es el rol de las tecnologías en el contexto actual, la pertinencia y tipo de formación en las áreas identificadas, así como la articulación y coherencia con el actual contexto socio-cultural de la región y el país. En síntesis, se debe terminar este proceso en aras de generar una propuesta de transformación en los contenidos de los posgrados de la FCSH en la UAC en aras de abordar el campo de la comunicación con mayor certeza y entendimiento de las interacciones actuales.

\subsection{Recomendaciones}

Es prudente aclarar que no se cuentan con recomendaciones profundas, se considera pertinente dejar una recomendación tentativa sobre los procesos educativos al interior de las especializaciones en cuestión. Si bien, como se estableció previamente, no hay fundamentos suficientes para realizar propuestas formales o certeras, los ahora egresados muestran unas inclinaciones sobre las áreas de trabajo que se proyectan dentro de su campo profesional, las cuales, independientemente de proporción o profundidad, exhiben la necesidad de explorar la relación campo-estudiantes-egresados y comprender las relaciones intersubjetivas consecuentes. Con base en ello, se estima encontrar una fuente de no solo robustecimiento, pero mejoramiento de la calidad educativa de las Especializaciones de la FCSH en la Universidad Autónoma del Caribe.

\section{REFERENCIAS}

Beltrán, C. (2016). Observatorio de medios y mediaciones como vínculo entre docencia e investigación. Opción, 32 (No. Especial 11), 75 - 87. Recuperado de https://dialnet.unirioja.es/descarga/articulo/5866898.pdf

Cadavid Bringe, A. y Gumucio-Dagron, A. (2014). Pensar desde la experiencia: Comunicación participativa en el Cambio Social. Colombia: Corporación Universitaria Minuto de Dios, UNIMINUTO. 
Gonzales, O. y Rosero, J. (2015). Abordajes de la Comunicación tomado desde la perspectiva del Interaccionismo y la Transmulti e Interdisplinariedad. XVII Congreso de Redcom (Argentina). La institucionalización de los debates, estudios e incidencia social del campo de la Comunicación. Recuperado de https://bit.ly/31rxDQU

Gumucio-Dagron, A. (2011). Comunicación para el cambio social: Clave de desarrollo participativo. Signo y Pensamiento, 30 (58), 26-39. Recuperado de https://www.redalyc.org/pdf/860/86020038002.pdf

Hernández, R., Fernández, C. y Baptista, P. (2015). Metodología de la Investigación: Sexta Edición. México: Editorial Mc Graw Hill.

Krummel, D. (1993). Bibliografías: sus objetivos y métodos. Traducciòn del inglés de Isabel Fonseca Ruiz. Madrid, etc.: Fundación Germán Sánchez Ruipérez; Madrid: Pirámide; Medellín: Colombia.

Múnera, P. (2010). Articulación Teórico-práctica de la Comunicación. Medellín, Colombia: Editorial Zuluaga.

Pérez, T. (2016). Sistematización de experiencias en contextos universitarios. Caracas, Venezuela: Universidad Nacional Abierta.

Piñeros, E. (2018). Estado del Arte de la Comunicación Organizacional en el Departamento del Meta-Colombia, a partir de los proyectos de grado en investigación registrados del año 2013 al año 2017 en las universidades presentes en el departamento. Villavicencio, Colombia: UNIMINUTO regional llanos.

Pita Fernández, S. y Pértegas Díaz, S. (2002). Investigación cuantitativa y cualitativa. CAD ATEN PRIMARIA, 9, 76-78. Recuperado de http://www.fisterra.com/mbe/investiga/cuanti cuali/cuanti cuali.asp

Popper, K (1989). La lógica de la investigación científica. Traducción de V. Sánchez de Zavala. Madrid: Tecnos.

Proyecto Educativo de Programa -PEP-. (2016). Especializaciones GCO y GDS. Barranquilla, Colómbia: Universidad Autónoma del Caribe.

Ramírez, D., Martínez, L. y Castellanos, O. (2012). Divulgación y difusión del conocimiento: las revistas científicas. Recuperado de https://bit.ly/30Fkbd5

UNESCO. (2012). Alfabetización mediática e informacional. Recuperado de https://bit.ly/3nis28V

Vasallo de Lopes, M.I. (1999). La investigación de la comunicación: cuestiones epistemológicas, teóricas y metodológicas. Diálogos de la Comunicación, 56. Recuperado de https://bit.ly/33u5V7V 
Villaseñor, I. (2008). Metodología para la elaboración de guías de fuentes de información. España: Universidad Complutense de Madrid. Recuperado de https://bit.ly/2DH4WHD

Yelitza, C. (2008). Teoría critico social. Documento en línea. Recuperado el 10 de diciembre de 2019, de https://bit.ly/3nlbhKy

\section{AUTORES}

\section{Javier Rosero Molina}

Comunicador Social-Periodista, Magister en Comunicación y Derechos Humanos, Especialista en Comunicación para el Desarrollo Social. Con enfoques en Comunicación y Cultura, Comunicación y Cambio Social, Espacio público. He desarrollado proyectos que apoyan el cambio social desde la comunicación interesándome en colectivos de comunicaciones, comunidades minoritarias y medios alternativos, entre otros. He trabajado con instituciones del sector privado y/o público, así como en Instituciones de Educación Superior desempeñándome con responsabilidad y ética, con el firme propósito de contribuir al bienestar colectivo y a promover un pensamiento más comprensivo sobre la comunicación.

ORCID ID: https://orcid.org/0000-0002-2368-5757

Google Académico: https://scholar.google.com/citations?user=5s0XyLUAAAAJ

\section{Darvis Rafael Rodriguez Chaparro}

Magister en Ciencias de la Comunicación y Profesional en Ingeniería de Sistemas, Docente tiempo completo en los Programas de Comunicación Social Periodismo y Comunicación Audiovisual, miembro activo del grupo de investigación Comunicación y Región de la Facultad de Ciencias Sociales y Humanas de la Universidad Autónoma del Caribe.

ORCID: https://orcid.org/0000-0001-8644-6828

Google Académico: https://scholar.google.es/citations?user=M5cVUzkAAAAJ

\section{Carlos Ramos Maldonado}

Comunicador Social - Periodista; magíster en Comunicación y NTICs y candidato a doctor de Ciencias Humanas en la Universidad del Zulia (Venezuela). Ex concejal de Barranquilla, ex congresista del Atlántico. Secretario de Educación (1992 -1993) y de Comunicaciones (2003) de la Alcaldía Distrital de Barranquilla. Catedrático de las universidades Autónoma del Caribe, Atlántico, Uninorte, ESAP y UNAD. Autor de los poemarios "Las mariposas son flores que juegan a la libertad", "Alas de la soledad" y "La ópera del profeta", y de las biografías noveladas "Merecumbé" y "Alicia se volvió canción". Decano de la Facultad de Ciencias Sociales y Humanas (2013 y 2018) y actual coordinador de las Especializaciones en Comunicación de la Universidad Autónoma del Caribe.

ORCID ID: https://orcid.org/0000-0002-7034-3389

Google Académico: https://bit.ly/3gLvGp2 